\title{
Clarifying haplotype ambiguity of NAT2 in multi-national cohorts
}

\section{Silvia Selinski ${ }^{1}$, Meinolf Blaszkewicz ${ }^{1}$, Jose A. G. Agundez ${ }^{2}$, Carmen Martinez ${ }^{2}$, Elena Garcia-Martin ${ }^{3}$, Jan G. Hengstler ${ }^{1}$, Klaus Golka ${ }^{1}$}

${ }^{1}$ Leibniz Research Centre for Working Environment and Human Factors (IfADo), Dortmund, Germany, ${ }^{2}$ Department of Pharmacology, University of Extremadura, Badajoz, Spain, ${ }^{3}$ Department of Biochemistry and Molecular Biology, University of Extremadura, Badajoz, Spain

\section{TABLE OF CONTENTS}

\author{
1. Abstract \\ 2. Introduction \\ 3. Materials and methods \\ 3.1. Subjects \\ 3.2. NAT2 genotyping \\ 3.3. Statistical analysis \\ 4. Results \\ 4.1. Clarifying haplotype ambiguity \\ 4.2. Investigating ethnic differences in controls \\ 4.3. Ethnic differences in haplotype distribution with respect to bladder cancer \\ 5. Discussion \\ 5.1. Haplotype assignment \\ 5.2. Haplotype distribution \\ 5.3. Haplotype implications \\ 5.4. Recent advances in NAT2 phenotype prediction: Reducing the genotype to one or two SNPS \\ 5.5. Summary \\ 6. Acknowledgements \\ 7. References
}

\section{ABSTRACT}

$\mathrm{N}$-Acetyltransferase 2 (NAT2) is the key enzyme in aromatic amine metabolism. NAT2 genotyping requires a subsequent determination of the haplotype pairs (formerly: alleles) to derive the acetylation status. The chromosomal phase of the single nucleotide polymorphisms (SNPs) is unclear for about $2 / 3$ of the genotypes. We investigated NAT2 genotypes of 1,234 bladder cancer cases and 2,207 controls from Germany, Hungary, Pakistan and Venezuela plus 696 further German cancer cases. We reconstructed NAT2 haplotypes using PHASE v2.1.1. We analysed if the variability of the NAT2 haplotypes affected the haplotype reconstruction. Furthermore, we compared population haplotype frequencies in three Caucasian control cohorts (German, Hungarian, Spanish), in Pakistanis and Venezuelans and the impact on bladder cancer. We conclude that a common haplotype reconstruction is feasible, enhances precision and reliability. Hungarian controls showed the largest intra-ethnic variability whereas the Pakistanis showed a haplotype distribution typical for Caucasians. The main differences could be observed for the slow haplotypes $* 5 \mathrm{~B}, * 6 \mathrm{~A}$ and $* 7 \mathrm{~B}$. The association of slow NAT2 genotypes with bladder cancer risk was most prominent in the Venezuelan study group.

\section{INTRODUCTION}

Human arylamine $N$-acetyltransferase 2 (NAT2) plays a key role in the metabolism of aromatic amines (13). Polymorphisms in the NAT2 gene were at first discovered as modifiers of blood and urine levels of antituberculosis drugs, in particular, of the ratio of free biologically active isoniazid compared to its mainly acetylated metabolites $(4,5)$.

Since then, numerous studies have analysed the impact of inter-individual variation in the acetylation capacity on drug efficacy and side effects as well as on cancer susceptibility especially focussing on exposure to carcinogenic NAT2 substrates such as aromatic and heterocyclic amines (2, 6-21). The role of NAT2 in cancer development is debated for a number of tumours (for review see 22); with respect to bladder cancer large studies and meta-analyses show an increased risk for slow acetylators, in particular, if they were exposed to aromatic amines or smoking $(6,15,23,24)$.

Inference of the NAT2 phenotype from the genotype is a non-trivial task as reflected by numerous studies dedicated to this issue $(13,14,25-36)$. Commonly a 
panel of seven single nucleotide polymorphisms (SNPs), in particular G191A (rs1801279), C282T (rs1041983), T341C (rs1801280), C481T (rs1799929), G590A (rs1799930), A803G (rs1208) and G857A (rs1799931), is used to determine slow and rapid NAT2 haplotypes (formerly: alleles) (31, 34, 37-41) according to the consensus nomenclature (33, 42-44, http://www.louisville.edu/medschool/pharmacology/NAT.html).

Besides simple prediction of slow and rapid acetylation capacity the distinct haplotypes of these seven SNPs are discussed with respect to velocity and substrates (23, $25,29-31,41,45-48)$. The difficulty in phenotype prediction arises with ambiguity of haplotype assignment as the standard PCR- and RFLP-based methods provide no phase information, i.e. which genetic variants occur together on the same chromosome. Several studies aimed to clarify this situation, however with increasing numbers of studies and, thus, genotypes the decision for the most likely haplotype pair, given the unphased genotype information, might be still difficult for less frequent haplotypes $(37-39,49)$.

In particular, substantial inter- and even intra-ethnic variation of the seven characteristic NAT2 SNPs is well known $(23,34,50-52)$ and assumed to result from population-specific selective pressures possibly associated with upcoming agriculture and the resulting change of diet and exposure to xenobiotics $(49,51,53-55)$ so that haplotype inference between populations might be problematic. Sabbagh and Darlu conclude that a violation of the assumed haplotype patterns, e.g. assuming a haplotype distribution typical for Caucasians, may lead to a bias in haplotype designation from unphased genotypes (56). So, haplotypes could not necessarily be inferred from the literature, especially in non-Caucasian populations due to variations in the linkage disequilibrium patterns, besides further NAT2 polymorphisms only relevant in a particular population, e.g. $(49,55)$.

These known population differences prompted us to analyse their impact on haplotype reconstruction and haplotype distributions in healthy controls as well as in bladder cancer cases of different Caucasian and non-Caucasian populations.

In this study, we reconstructed haplotype pairs from 4,337 subjects (Germans, Hungarians, Pakistanis, Venezuelans) evaluating the effect of different haplotype patterns and compared the reconstruction with sequenced NAT2 haplotypes from a Spanish cohort (37). As inter- and intra-ethnic differences are usually considered in terms of single SNPs instead of haplotypes, we compared population frequencies of the haplotype distribution in three European control cohorts (German, Hungarian, Spanish), in Pakistanis and Venezuelans and evaluated potential differences between German, Hungarian, Pakistani and Venezuelan bladder cancer cases and controls.

\section{MATERIALS AND METHODS}

\subsection{Subjects}

Genotypes of 4,337 subjects from Germany $(\mathrm{n}=3408)$, Hungary $(\mathrm{n}=333)$, Pakistan $(\mathrm{n}=331)$ and Venezuela $(n=265)$ were included in the haplotype reconstruction. The German study group comprised 1,212 healthy controls, 140 persons with coxarthrosis or gonarthrosis, 579 persons with a connective tissue disease, 781 urinary bladder cancer cases, 309 head and neck cancer cases, 194 renal cell carcinoma cases and 193 colon cancer cases who had visited the Central Unit Clinical Occupational Medicine of our institute (IfADo) for different purposes or who were members of study cohorts in different hospitals. The Hungarian study group consisted of 61 hospital-based controls and 272 urinary bladder cancer cases, the Pakistani study group encompassed 225 population based and hospital-based controls and 106 urinary bladder cancer cases and the Venezuelan study group comprised 75 hospital-based controls and 190 urinary bladder cancer cases. Thus, we obtained 1,688 controls and 2,649 subjects from the different case groups. German patients with cox- or gonarthrosis or connective tissue diseases $(n=719)$ were used as urinary bladder cancer controls as the genotype distribution showed no deviation from the German healthy controls. The Spanish study group consists of 1,312 healthy controls as described by Agundez et al. (37). The sample collection was approved by the local Ethics Committee and by the IRB (institutional review board).

\subsection{NAT2 genotyping}

Each subject had donated $10 \mathrm{ml}$ EDTA blood. DNA was extracted out of leukocytes using standard methods (QIAamp DNA Blood Maxikit, Hilden, Germany) and stored at $+4^{\circ} \mathrm{C}$. NAT2 genotyping was performed using PCR- and RFLP-based standardized methods (41, 57, 58). A total of seven SNPs, which were adequate to genotype Caucasians for NAT2 (57), were investigated, namely rs1801279 (G191A), rs1041983 (C282T), rs1801280 (T341C), rs1799929 (C481T), rs1799930 (G590A), rs1208 (A803G) and rs1799931 (G857A). Leukocyte DNA was isolated from a sample of human blood. Amplification of two fragments of DNA with 442 and 559 bp (base pairs) was achieved by means of PCR (polymerase chain reaction). The amplicon from the first PCR was cleaved using three different restriction enzymes, and that of the second PCR with four different restriction enzymes. After subsequent gel electrophoresis with the addition of ethidium bromide, the various DNA fragments were detected in UV light. The results were documented by photography, and the alleles were assigned according to an evaluation scheme (57).

\subsection{Statistical analysis}

The haplotype analysis was performed using the program PHASE v2.1.1 (59-61). The approach underlying PHASE is a Bayesian haplotype reconstruction method using coalescent-based models to improve the accuracy of haplotypes for unrelated individuals (62). We used the default model for recombination rate variation (63) to estimate the individual haplotype pairs, their probability as well as the sample haplotype frequencies and applied the implemented permutation test for differences in haplotype frequencies that takes the uncertainty of the haplotype reconstruction into account. We tested for differences between German, Hungarian, Pakistani and Venezuelan controls as well as for differences between cases and controls separately in each of the bladder cancer case- 
control series. We performed five independent runs with 1,000 main iterations, 1,000 burn-in iterations and a thinning interval of 1 . For the permutation test we started with 100 iterations. We chose the best run showing the maximum consistency across five runs and repeated the analysis with the same settings but increased the permutations to 10,000 for the test. Slow and rapid NAT2 genotypes were deduced according to the consensus NAT2 gene nomenclature assuming *4, *12 and *13 haplotypes as rapid (44). We calculated odds ratios (OR), 95\% confidence intervals $(95 \%$ $\mathrm{CI}$ ) and $\mathrm{P}$ values of the exact Fisher test of differences between bladder cancer cases and controls separately for each study group using the SAS/STAT software, version 9.2 (SAS Institute Inc., Cary, USA).

\section{RESULTS}

We reconstructed the haplotype pairs to clarify the chromosomal phase and estimated the sample frequencies to compare the different control groups and to evaluate differences in the modulating effect of NAT2 on bladder cancer in different populations.

\subsection{Clarifying haplotype ambiguity}

First, we clarified the ambiguous genotypes in the maximum data set of 1,688 healthy subjects and 2,649 cancer cases (mainly urothelial bladder cancer) from Germany, Hungary, Pakistan and Venezuela (Table 1). We discovered 38 different diplotypes, for 22 of them the phase was not clear. Considering the rather frequent genotypes with a frequency of $>1 \%$ in the controls eight of 12 genotypes could not be determined without phase ambiguity $(67 \%)$, for 1,096 out of 1,593 controls the haplotype pair remained unclear from PCR/RFLP methods (69\%; all controls: 1,151 out of $1,688,68 \%$ ). This ambiguity could be clarified completely by haplotype reconstruction with a probability of the reconstructed haplotype pairs of $\mathrm{P}>0.93$. In particular, all but one haplotype pair had a probability of $\mathrm{P}>0.98$, most of them were almost sure $(\mathrm{P}=1.00)$.

Comparing the haplotype reconstruction to earlier results, we discovered a single deviation to the haplotype reconstruction and the verification of haplotypes by sequencing in case of the unphased genotype $\mathrm{G} / \mathrm{G}, \mathrm{C} / \mathrm{C}$, $\mathrm{C} / \mathrm{C}, \mathrm{C} / \mathrm{T}, \mathrm{G} / \mathrm{G}, \mathrm{A} / \mathrm{G}, \mathrm{G} / \mathrm{G}(0021010 ; * 5 \mathrm{~A} / * 5 \mathrm{C}$ or $* 5 \mathrm{~B} / * 5 \mathrm{D})$. We obtained as the most likely haplotype pair $* 5 \mathrm{~A} / * 5 \mathrm{C}$ with $\mathrm{P}=0.992(\mathrm{n}=3)$ whereas sequencing yielded $* 5 \mathrm{~B} / * 5 \mathrm{D}$ in a Spanish cohort in accordance with the former haplotype reconstruction also with PHASE v2.1.1 and similar models and adjustments $(* 5 \mathrm{~A} / * 5 \mathrm{C}$ less probable with $\mathrm{P}=0.09, * 5 \mathrm{~B} / * 5 \mathrm{D}$ more likely with $\mathrm{P}=0.91$ ) (37). Further deviations from this study could not be determined, in particular, those genotypes for which PHASE yielded other results than sequencing in the Spanish study group were not present in the current data.

\subsection{Investigating ethnic differences in controls}

As the German cox- and gonarthrosis patients and persons with a connective tissue disease had the same haplotype distribution as the respective controls $(\mathrm{P}=0.3500)$ we used them as additional controls for ethnic differences
(Table 2, 3) and bladder cancer (Table 4, 5). The haplotype sample frequencies in German, Hungarian, Pakistani and Venezuelan controls showed clear differences $(\mathrm{P}=0.0001$; Table 2). Comparing these results to the Spanish haplotype distribution further discrepancies became apparent. In particular, the most frequent haplotypes $* 4, * 5 B, * 6 \mathrm{~A}$ and $* 7 \mathrm{~B}$ showed a considerable inter- and intra-ethnic variability (Table 2 ). The rapid *4 haplotype was most frequent in Hungarians (28\%) and Venezuelans (27\%) whereas Germans and Spaniards (both 22\%) showed the lowest frequencies. The frequency of $* 4$ in Pakistani controls was similar to the one in Spanish and German controls $(24 \%)$.

The slow $* 5 \mathrm{~B}$ haplotype varied most among Europeans (25\% in Hungarians, $43 \%$ in Spaniards). *5B was also most frequent in the German (41\%) and Pakistani $(40 \%)$ controls as well as in the Venezuelans though in the latter $* 5 \mathrm{~B}$ was clearly less frequent (32\%). Similarly the slow $* 6 \mathrm{~A}$ haplotype exhibited the most differences between the European study groups (Spain 25\%, Germany 29\%, Hungary 37\%) with the lowest frequencies in Venezuelans (22\%). Again, the Pakistani haplotype frequency (28\%) was similar to the German one. The slow *7B haplotype was most frequent in Venezuelans $(9 \%)$ whereas its frequency in Pakistanis $(4 \%)$, Germans $(3 \%)$ and Spaniards $(1 \%)$ was rather low. The Hungarian controls showed again notable deviations from the other Europeans (6\%). Remarkably, in the Spanish cohort several of the *14 haplotypes that are rare in Caucasians could be verified. The Venezuelan controls exhibited also a relatively high frequency of the $* 14 \mathrm{~B}$ haplotype $(0.26 \%)$.

Discriminating only between rapid and slow haplotypes yielded a similar high percentage of slow haplotypes that was highest among the German controls and lowest in Venezuelans (Germany 77\%, Pakistan 76\%, Spain $75 \%$, Hungary $71 \%$, Venezuela $68 \%$ ). Hence slow acetylators are most common in German controls (60\%) and less common in Venezuelans (45\%, $\mathrm{P}=0.0129$ all control groups, Table 3). The differences between the German, Hungarian, Pakistani and Spanish controls are less relevant $(\mathrm{P}=0.3550$ excluding the Venezuelan controls).

Separate haplotype reconstruction for each study group did not yield different results. In particular, haplotypes sample frequencies varied at most 0.01 percent points, except for the Venezuelan controls that showed deviation of at most 0.2 percent points.

\subsection{Ethnic differences in haplotype distribution with respect to bladder cancer}

Finally, we investigated the impact of NAT2 haplotypes on bladder cancer risk in the German, Hungarian, Pakistani and Venezuelan study groups (Table $4,5)$. Differences between cases and controls could only be confirmed for the Venezuelan study group $(\mathrm{P}=0.0006)$ whereas German, Hungarian and Pakistani bladder cancer cases showed no significant alterations in haplotype frequency (Table 4). In particular, the Venezuelan controls 
Table 1. NAT2 genotyping yields about $2 / 3$ ambiguous results that could be clarified completely by haplotype reconstruction

\begin{tabular}{|c|c|c|c|c|c|c|c|c|}
\hline & \multicolumn{5}{|c|}{ Haplotype pair } & \multicolumn{3}{|c|}{ Frequency } \\
\hline Observed diplotype $^{1}$ & Haplotype1 & Haplotype 2 & Haplotype 1 & Haplotype 2 & P value & $\mathrm{N}$ total & Controls & Cancer cases \\
\hline 0000000 & GCTCGAG & GCTCGAG & $* 4$ & $* 4$ & 1.0 & 216 & $5.21 \%$ & $4.83 \%$ \\
\hline 0011000 & GCTCGAG & GCCTGAG & $* 4$ & $* 5 \mathrm{~A}$ & 1.0 & 65 & $1.18 \%$ & $1,70 \%$ \\
\hline 0011010 & GCTCGAG & GCCTGGG & $* 4$ & $* 5 \mathrm{~B}$ & 0.998 & 803 & $18.48 \%$ & $18,54 \%$ \\
\hline 0010010 & GCTCGAG & GCCCGGG & $* 4$ & $* 5 \mathrm{C}$ & 1.0 & 27 & $0.77 \%$ & $0.53 \%$ \\
\hline 0100100 & GCTCGAG & GTTCAAG & $* 4$ & $* 6 \mathrm{~A}$ & 1.0 & 550 & $11.97 \%$ & $13,14 \%$ \\
\hline 0000100 & GCTCGAG & GCTCAAG & $* 4$ & $* 6 \mathrm{~B}$ & 1.0 & 2 & $0.06 \%$ & $0.04 \%$ \\
\hline 0100001 & GCTCGAG & GTTCGAA & $* 4$ & $* 7 \mathrm{~B}$ & 1.0 & 66 & $2.25 \%$ & $1,06 \%$ \\
\hline 0000010 & GCTCGAG & GCTCGGG & $* 4$ & $* 12 \mathrm{~A}$ & 1.0 & 9 & $0.41 \%$ & $0.08 \%$ \\
\hline 0100000 & GCTCGAG & GTTCGAG & $* 4$ & $* 13 \mathrm{~A}$ & 1.0 & 2 & $0.12 \%$ & $0.00 \%$ \\
\hline 0022000 & GCCTGAG & GCCTGAG & $* 5 \mathrm{~A}$ & $* 5 \mathrm{~A}$ & 1.0 & 3 & $0.00 \%$ & $0.11 \%$ \\
\hline 0022010 & GCCTGAG & GCCTGGG & $* 5 \mathrm{~A}$ & $* 5 \mathrm{~B}$ & 1.0 & 73 & $0.95 \%$ & $2,15 \%$ \\
\hline 0021010 & GCCTGAG & GCCCGGG & $* 5 \mathrm{~A}$ & $* 5 \mathrm{C}$ & 0.992 & 3 & $0.00 \%$ & $0.11 \%$ \\
\hline 0111100 & GCCTGAG & GTTCAAG & $* 5 \mathrm{~A}$ & $* 6 \mathrm{~A}$ & 1.0 & 64 & $1.42 \%$ & $1,51 \%$ \\
\hline 0111001 & GCCTGAG & GTTCGAA & $* 5 \mathrm{~A}$ & $* 7 \mathrm{~B}$ & 1.0 & 5 & $0.06 \%$ & $0.15 \%$ \\
\hline 0022020 & GCCTGGG & GCCTGGG & $* 5 \mathrm{~B}$ & $* 5 \mathrm{~B}$ & 1.0 & 645 & $14.93 \%$ & $14,84 \%$ \\
\hline 0021020 & GCCTGGG & GCCCGGG & $* 5 \mathrm{~B}$ & $* 5 \mathrm{C}$ & 1.0 & 62 & $1.18 \%$ & $1,59 \%$ \\
\hline 0111110 & GCCTGGG & GTTCAAG & $* 5 \mathrm{~B}$ & $* 6 \mathrm{~A}$ & 1.0 & 1,035 & $24.76 \%$ & $23,29 \%$ \\
\hline 0111120 & GCCTGGG & GTTCAGG & $* 5 \mathrm{~B}$ & $* 6 \mathrm{C}$ & 1.0 & 2 & $0.00 \%$ & $0.08 \%$ \\
\hline 0111011 & GCCTGGG & GTTCGAA & $* 5 \mathrm{~B}$ & $* 7 \mathrm{~B}$ & 1.0 & 122 & $2.78 \%$ & $2,83 \%$ \\
\hline 0011020 & GCCTGGG & GCTCGGG & $* 5 \mathrm{~B}$ & $* 12 \mathrm{~A}$ & 0.999 & 30 & $0.59 \%$ & $0.76 \%$ \\
\hline 0111010 & GCCTGGG & GTTCGAG & $* 5 \mathrm{~B}$ & $* 13 \mathrm{~A}$ & 0.997 & 7 & $0.36 \%$ & $0.04 \%$ \\
\hline 1111010 & GCCTGGG & ATTCGAG & $* 5 \mathrm{~B}$ & $* 14 \mathrm{~B}$ & 0.995 & 1 & $0.06 \%$ & $0.00 \%$ \\
\hline 1022020 & GCCTGGG & ACCTGGG & $* 5 \mathrm{~B}$ & $* 14 \mathrm{C}$ & 1.0 & 1 & $0.06 \%$ & $0.00 \%$ \\
\hline 0020020 & GCCCGGG & GCCCGGG & $* 5 \mathrm{C}$ & $* 5 \mathrm{C}$ & 1.0 & 8 & $0.12 \%$ & $0.23 \%$ \\
\hline 0110110 & GCCCGGG & GTTCAAG & $* 5 \mathrm{C}$ & $* 6 \mathrm{~A}$ & 1.0 & 45 & $0.65 \%$ & $1 \%$ \\
\hline 0110011 & GCCCGGG & GTTCGAA & $* 5 \mathrm{C}$ & $* 7 \mathrm{~B}$ & 1.0 & 4 & $0.12 \%$ & $0.08 \%$ \\
\hline 0010020 & GCCCGGG & GCTCGGG & $* 5 \mathrm{C}$ & $* 12 \mathrm{~A}$ & 1.0 & 1 & $0.06 \%$ & $0.00 \%$ \\
\hline 0200200 & GTTCAAG & GTTCAAG & $* 6 \mathrm{~A}$ & $* 6 \mathrm{~A}$ & 1.0 & 376 & $8.12 \%$ & $9,02 \%$ \\
\hline 0200210 & GTTCAAG & GTTCAGG & $* 6 \mathrm{~A}$ & $* 6 \mathrm{C}$ & 1.0 & 2 & $0.00 \%$ & $0.08 \%$ \\
\hline 0200101 & GTTCAAG & GTTCGAA & $* 6 \mathrm{~A}$ & $* 7 \mathrm{~B}$ & 1.0 & 73 & $2.07 \%$ & $1,43 \%$ \\
\hline 0100110 & GTTCAAG & GCTCGGG & $* 6 \mathrm{~A}$ & $* 12 \mathrm{~A}$ & 0.934 & 13 & $0.41 \%$ & $0.23 \%$ \\
\hline 0101110 & GTTCAAG & GCTTGGG & $* 6 \mathrm{~A}$ & $* 12 \mathrm{C}$ & 0.983 & 1 & $0.00 \%$ & $0.04 \%$ \\
\hline 0200100 & GTTCAAG & GTTCGAG & $* 6 \mathrm{~A}$ & $* 13 \mathrm{~A}$ & 1.0 & 6 & $0.06 \%$ & $0.19 \%$ \\
\hline 1200100 & GTTCAAG & ATTCGAG & $* 6 \mathrm{~A}$ & $* 14 \mathrm{~B}$ & 1.0 & 2 & $0.06 \%$ & $0.04 \%$ \\
\hline 0200002 & GTTCGAA & GTTCGAA & $* 7 \mathrm{~B}$ & $* 7 \mathrm{~B}$ & 1.0 & 7 & $0.41 \%$ & $0.00 \%$ \\
\hline 0100011 & GTTCGAA & GCTCGGG & $* 7 \mathrm{~B}$ & $* 12 \mathrm{~A}$ & 0.999 & 2 & $0.12 \%$ & $0.00 \%$ \\
\hline 0200001 & GTTCGAA & GTTCGAG & $* 7 \mathrm{~B}$ & $* 13 \mathrm{~A}$ & 1.0 & 3 & $0.12 \%$ & $0.04 \%$ \\
\hline 2011010 & ACTCGAG & ACCTGGG & $* 14 \mathrm{~A}$ & $* 14 \mathrm{C}$ & 1.0 & 1 & $0.06 \%$ & $0.00 \%$ \\
\hline & & & & & Sum & 4,337 & 1,688 & 2,649 \\
\hline
\end{tabular}

$\mathrm{N}$ total: total number of observed diplotypes, 0 : homozygous reference, 1 : heterozygous, 2 : homozygous variant. ${ }^{1}$ Observed diplotypes are shown as the number of variant alleles at each locus (G191A, C282T, T341C, C481T, G590A, A803G, G857A). Ambiguous diplotypes with at least two heterozygous loci are highlighted grey. Considering diplotypes with a frequency in controls of $>1 \%$ for $69 \%$ of all control subjects and eight of twelve diplotypes the corresponding haplotype pairs are not clear.

exhibited more rapid $* 4(27 \%$ controls vs. $22 \%$ cases $)$, $* 12 \mathrm{~A} \quad(2.4 \% \quad v s . \quad 0.05 \%)$ and $* 13 \mathrm{~A} \quad(2.5 \%$ vs. $0 \%)$ haplotypes and slow *7B (9\% vs. 3\%) haplotypes whereas the slow *5A (2\% vs. $4 \%), * 5 \mathrm{~B}(32 \%$ vs. $46 \%)$ and $* 5 \mathrm{C}$ $(1.6 \%$ vs. $2.7 \%)$ haplotypes are more prominent in Venezuelan cases (Table 4). Discriminating only between rapid and slow haplotypes yielded an elevated risk for the slow acetylators $(68 \%$ vs. $78 \%$ slow haplotypes, $\mathrm{P}=0.0258$, $\mathrm{OR}=1.68,95 \% \quad \mathrm{CI}=1.06-2.70 ; \quad 45 \%$ vs. $59 \%$ slow acetylators, $\mathrm{P}=0.0493$, $\mathrm{OR}=1.72$, see Table 5).

Differences between controls and bladder cancer cases were also present in Hungarians (more frequent in controls: *4, *6A; more frequent in cases: $* 5 \mathrm{~B}, * 5 \mathrm{C}$; $\mathrm{P}=0.1524$ ) and Pakistanis (more frequent in controls: $* 5 \mathrm{~B}$, *7B; more frequent in cases: $* 6 \mathrm{~A} ; \mathrm{P}=0.1591$; see Table 4) but these deviations were not significant. The frequency of the slow acetylators was not elevated in Pakistani cases (76\% vs. $75 \%$ slow haplotypes, $\mathrm{P}=0.8470, \mathrm{OR}=0.95,95 \%$ $\mathrm{CI}=0.64-1.41$; slow acetylators $\mathrm{P}=0.7039$ ) but more slow haplotypes could be found Hungarian cases (71\% vs. $78 \%$ slow haplotypes, $\mathrm{P}=0.1241$, $\mathrm{OR}=1.42,95 \% \mathrm{CI}=0.88-2.25$; slow acetylators $\mathrm{P}=0.2114$ ). The largest study group from
Germany showed negligible differences with respect to the rapid $* 4(22 \%$ vs. $23 \%)$ and the slow $* 5 \mathrm{~B}(41 \%$ vs. $39 \%)$ and *6A $(29 \%$ vs. $30 \%)$ haplotypes $(\mathrm{P}=0.2254)$ and with respect to the frequency of slow haplotypes combined that were present in $77 \%$ and $76 \%$ of the German controls and cases, respectively $(\mathrm{P}=0.5218, \mathrm{OR}=0.96,95 \% \mathrm{CI}=0.83$ 1.10; slow acetylators $\mathrm{P}=0.8478$ ). Remarkably, the Venezuelan bladder cancer cases showed a much higher frequency of the slow $* 5 \mathrm{~B}$ haplotype $(46 \%)$ and lower $* 6 \mathrm{~A}$ frequency $(23 \%)$ than all other study groups (Hungary and Pakistan ca. 33\% each, Germany $39 \%$ and $33 \%$ ) while the frequency of the rapid haplotypes was comparable (22$25 \%)$.

\section{DISCUSSION}

NAT2 is known to be highly polymorphic in different populations $(23,34,50,52)$. The phase II enzyme is involved in the activation and inactivation, respectively, of many drugs and xenobiotics $(1-3,18,20,23,25,35,45)$ and thus discussed as susceptibility factor for a number of tumours (see 22 for review). Several studies and metaanalyses identified NAT2 as risk factor for urinary bladder 
Table 2. The haplotype frequencies of NAT2 differ clearly between German, Hungarian, Spanish, Pakistani and Venezuelan controls

\begin{tabular}{|c|c|c|c|c|c|c|c|c|c|c|c|c|}
\hline \multirow{2}{*}{\multicolumn{2}{|c|}{ Haplotype }} & \multicolumn{2}{|l|}{ Total } & \multicolumn{2}{|c|}{ Germany } & \multicolumn{2}{|l|}{ Hungary } & \multicolumn{2}{|l|}{ Pakistan } & \multicolumn{2}{|c|}{ Venezuela } & \multirow{2}{*}{$\begin{array}{l}\text { Spain } \\
\text { Freq }\end{array}$} \\
\hline & & E(Freq) & S.E. & E(Freq) & S.E. & E(Freq) & S.E. & E(Freq) & S.E. & E(Freq) & S.E. & \\
\hline GCTCGAG & $* 4$ & $22.75 \%$ & 0.000219 & $21.97 \%$ & 0.000247 & $27.86 \%$ & 0.001001 & $24.42 \%$ & 0.000650 & $27.10 \%$ & 0.000844 & $22.0 \%$ \\
\hline GCCTGAG & $* 5 \mathrm{~A}$ & $2.26 \%$ & 0.000201 & $2.27 \%$ & 0.000231 & $2.47 \%$ & 0.001002 & $2.24 \%$ & 0.000650 & $2.12 \%$ & 0.000664 & $1.0 \%$ \\
\hline GCCTGGG & $* 5 \mathrm{~B}$ & $39.88 \%$ & 0.000207 & $41.10 \%$ & 0.000234 & $25.40 \%$ & 0.001033 & $39.76 \%$ & 0.000650 & $32.34 \%$ & 0.000856 & $43.0 \%$ \\
\hline GCCCGGG & $* 5 \mathrm{C}$ & $1.50 \%$ & 0.000031 & $1.48 \%$ & 0.000035 & $0.82 \%$ & 0.000257 & $1.78 \%$ & 0.000070 & $1.58 \%$ & 0.000005 & $0.7 \%$ \\
\hline GCCCGAG & $* 5 \mathrm{D}$ & $0.00 \%$ & 0.000029 & $0.00 \%$ & 0.000033 & n.p. & n.p. & $0.00 \%$ & 0.000070 & n.p. & n.p. & $0.3 \%$ \\
\hline GTCTGGG & $* 5 \mathrm{G}$ & $0.00 \%$ & 0.000039 & $0.00 \%$ & 0.000030 & n.p. & n.p. & n.p. & n.p. & $0.00 \%$ & 0.000324 & $0.0 \%$ \\
\hline GTTCAAG & $* 6 \mathrm{~A}$ & $28.68 \%$ & 0.000105 & $29.15 \%$ & 0.000119 & $36.88 \%$ & 0.000258 & $27.78 \%$ & 0.000099 & $22.36 \%$ & 0.000456 & $25.0 \%$ \\
\hline GCTCAAG & $* 6 \mathrm{~B}$ & $0.04 \%$ & 0.000000 & $0.05 \%$ & 0.000000 & n.p. & n.p. & n.p. & n.p. & n.p. & n.p. & $2.0 \%$ \\
\hline GTTCAGG & $* 6 \mathrm{C}$ & $0.03 \%$ & 0.000102 & $0.03 \%$ & 0.000115 & n.p. & n.p. & $0.00 \%$ & 0.000099 & $0.01 \%$ & 0.000456 & $0.1 \%$ \\
\hline GTTCGAA & $* 7 \mathrm{~B}$ & $3.68 \%$ & 0.000011 & $3.00 \%$ & 0.000008 & $5.74 \%$ & 0.000257 & $4.00 \%$ & 0.000001 & $9.47 \%$ & 0.000083 & $1.0 \%$ \\
\hline GCTCGGG & $* 12 \mathrm{~A}$ & $0.78 \%$ & 0.000217 & $0.71 \%$ & 0.000247 & $0.83 \%$ & 0.001035 & $0.02 \%$ & 0.000650 & $2.38 \%$ & 0.000789 & $2.0 \%$ \\
\hline GTTCGAG & $* 13 \mathrm{~A}$ & $0.29 \%$ & 0.000071 & $0.13 \%$ & 0.000048 & $0.00 \%$ & 0.000257 & n.p. & n.p. & $2.37 \%$ & 0.000560 & $0.3 \%$ \\
\hline ACTCGAG & $* 14 \mathrm{~A}$ & $0.02 \%$ & 0.000000 & $0.03 \%$ & 0.000000 & n.p. & n.p. & n.p. & n.p. & n.p. & n.p. & $0.4 \%$ \\
\hline ATTCGAG & $* 14 \mathrm{~B}$ & $0.04 \%$ & 0.000057 & $0.03 \%$ & 0.000033 & n.p. & n.p. & n.p. & n.p. & $0.26 \%$ & 0.000442 & $0.0 \%$ \\
\hline ACCTGGG & $* 14 \mathrm{C}$ & $0.04 \%$ & 0.000035 & $0.05 \%$ & 0.000000 & n.p. & n.p. & n.p. & n.p. & $0.01 \%$ & 0.000442 & $0.5 \%$ \\
\hline ATTCAAG & $* 14 \mathrm{D}$ & $0.00 \%$ & 0.000027 & $0.00 \%$ & 0.000033 & n.p. & n.p. & n.p. & n.p. & n.p. & n.p. & $0.2 \%$ \\
\hline
\end{tabular}

Total: German, Hungarian, Pakistani and Venezuelan controls, E(Freq): Estimated frequency, S.E.: Standard error, Freq: Frequency, n.p.: not present, Spain: Haplotypes were verified by sequencing. $1 \%$ of the sample are haplotypes not present in the table $(* 12 \mathrm{C} 0.7 \% ; * 14 \mathrm{G} 0.2 \% ; * 12 \mathrm{~B} 0.1 \% ; * 5 \mathrm{E}, * 14 \mathrm{E}, * 14 \mathrm{~F}, * 14 \mathrm{I} 0.04 \%$ each). Frequent haplotypes with a frequency of $>5 \%$ in at least one study groups are highlighted grey, Differences in haplotype distribution between German, Hungarian, Pakistani and Venezuelan controls: $\mathrm{p}=0.0001(10,000$ iterations $)$

Table 3. The acetylation status frequencies differ clearly between German, Hungarian, Spanish, Pakistani and Venezuelan controls

\begin{tabular}{|l|l|l|l|l|l|}
\hline & \multicolumn{3}{|c|}{ Study group } \\
\hline Acetylation status & Germany & Hungary & Pakistan & Venezuela \\
\hline Slow & $59.55 \%$ & $50.82 \%$ & $56.00 \%$ & $45.26 \%$ & $5.79 \%$ \\
\hline Intermediate & $35.27 \%$ & $40.98 \%$ & $39.11 \%$ & $56.02 \%$ \\
\hline Rapid & $5.18 \%$ & $4.89 \%$ & $37.96 \%$ & $6.95 \%$ \\
\hline N & 1,931 & 225 & 190 & 1,312 \\
\hline
\end{tabular}

Chi-square test including the Venezuelan controls $\mathrm{P}=0.0129$, Chi-square test excluding the Venezuelan controls $\mathrm{P}=0.3550$

cancer $(6,15,23,24,64)$ especially in smokers $(20$, 65-67) and persons occupationally exposed to bladder carcinogens as aromatic amines (9-17, 68-71).

Incidences of urinary bladder cancer vary substantially between different populations due to differences in exogenous and endogenous risk factors. Age-standardised incidence rates vary for the present populations from 2.6/100,000 for Venezuela to $11.6 / 100,000$ for Germany (Hungary: 11.5, Pakistan 3.5) (ASR(W) incidences from IARC GLOBOCAN 2008 http://globocan.iarc.fr). Slow NAT2 status, especially in presence of bladder carcinogens, is a well-known risk factor for urinary bladder cancer that is highly variable between and within ethnicities (50). Recent studies report at most small to moderate bladder cancer risks for slow acetylators ranging from 1.01 (New England study (6)) to 1.15 (genome-wide study on Caucasians (24)) and 1.40-1.45 (meta-analysis (23) and Spanish bladder cancer study (64)) if smoking is not taken into account. The present data showed an odds ratio of 1.04 for all study groups combined with an elevated bladder cancer risk for slow acetylators in Venezuelans and Hungarians but not in the German and Pakistani study group. Taking different distributions of gender, age and smoking habits into account would yield more reliable results but a certain variation in bladder cancer risk is likely to remain due to the variation of further exogenous and endogenous risk factors.

\subsection{Haplotype assignment}

Studies on NAT2 impact are hampered by unclear haplotype assignment as unphased genotypes of seven SNPs result in more than one possible haplotype pair if more than one of these seven SNPs is heterozygous. Theoretically, for $n$ heterozygous SNPs there are $2^{(n-1)}$ possible haplotype pairs, though in fact some of them are rare or have never been observed. For instance, the NAT2 haplotype pair $* 5 \mathrm{~B} / * 6 \mathrm{~A}$ results in an unphased genotype with five heterozygous SNPs (*5B: T341C, C481T, A803G; *6A: C282T, G590A) leading to 16 possible haplotype pairs. Moreover, allele frequencies and thus haplotype frequencies and linkage disequilibrium patterns vary across populations $(50,72)$ so identifying the genotypic phase is not a trivial task and has to be handled with care across populations (56). The present results suggest that for populations that do not vary extremely in their haplotype structure a common reconstruction is feasible and check-ups within the sub-datasets from the same population can be done with less precision. In particular, we observe that also infrequent unphased genotypes result in the same reconstructed haplotype pairs even if the different populations are considered separately.

However, sequencing data showed that the same unphased genotype may in fact result in different haplotype pairs. This insecurity is reflected partially in the probability of the reconstructed haplotype pairs, so it is highly recommended to consider and record this measure of confidence in the estimate. In general, for haplotype 
Table 4. Estimated sample frequency of NAT2 haplotypes in bladder caner cases and controls in four study groups from Germany, Hungary, Pakistan and Venezuela

\begin{tabular}{|c|c|c|c|c|c|c|c|}
\hline \multirow[t]{2}{*}{ Study group } & \multirow{2}{*}{ Haplotype } & \multirow{2}{*}{$\begin{array}{l}\text { NAT2 allele } \\
\text { designation }\end{array}$} & \multicolumn{2}{|l|}{ Controls } & \multicolumn{2}{|l|}{ Cases } & \multirow[b]{2}{*}{ Frequency in the sample } \\
\hline & & & $E$ (freq) & S.E. & $E$ (freq) & S.E. & \\
\hline \multicolumn{8}{|l|}{ Germany } \\
\hline 781 cases & GCTCGAG & $* 4$ & $21.97 \%$ & 0.0002 & $23.17 \%$ & 0.0004 & 1211 \\
\hline 1,931 controls & GCCTGAG & $* 5 \mathrm{~A}$ & $2.27 \%$ & 0.0002 & $2.51 \%$ & 0.0003 & 126 \\
\hline \multirow[t]{13}{*}{$\mathrm{P}=0.2254$} & GCCTGGG & $* 5 \mathrm{~B}$ & $41.10 \%$ & 0.0002 & $38.72 \%$ & 0.0003 & 2193 \\
\hline & GCCCGGG & $* 5 \mathrm{C}$ & $1.48 \%$ & 0.0000 & $1.86 \%$ & 0.0000 & 86 \\
\hline & GTCTGGG & $* 5 \mathrm{G}$ & $0.00 \%$ & 0.0001 & $0.00 \%$ & 0.0000 & 0 \\
\hline & GTTCAAG & $* 6 \mathrm{~A}$ & $29.14 \%$ & 0.0002 & $30.27 \%$ & 0.0002 & 1599 \\
\hline & GCTCAAG & $* 6 \mathrm{~B}$ & $0.05 \%$ & 0.0000 & $0.00 \%$ & 0.0000 & 2 \\
\hline & GTTCAGG & $* 6 \mathrm{C}$ & $0.03 \%$ & 0.0001 & $0.01 \%$ & 0.0002 & 1 \\
\hline & GTTCGAA & $* 7 \mathrm{~B}$ & $3.00 \%$ & 0.0000 & $3.01 \%$ & 0.0000 & 163 \\
\hline & GCTCGGG & $* 12 \mathrm{~A}$ & $0.71 \%$ & 0.0002 & $0.45 \%$ & 0.0004 & 34 \\
\hline & GTTCGAG & $* 13 \mathrm{~A}$ & $0.14 \%$ & 0.0001 & $0.00 \%$ & 0.0000 & 5 \\
\hline & ACTCGAG & $* 14 \mathrm{~A}$ & $0.03 \%$ & 0.0000 & $0.00 \%$ & 0.0000 & 1 \\
\hline & ATTCGAG & $* 14 \mathrm{~B}$ & $0.01 \%$ & 0.0001 & $0.00 \%$ & 0.0000 & 0 \\
\hline & ACCTGGG & $* 14 \mathrm{C}$ & $0.05 \%$ & 0.0000 & $0.00 \%$ & 0.0000 & 2 \\
\hline & ATTCAAG & $* 14 \mathrm{D}$ & $0.01 \%$ & 0.0001 & $0.00 \%$ & 0.0000 & 1 \\
\hline \multicolumn{8}{|l|}{ Hungary } \\
\hline 272 cases & GCTCGAG & $* 4$ & $27.85 \%$ & 0.0012 & $20.82 \%$ & 0.0010 & 147 \\
\hline 61 controls & GCCTGAG & $* 5 \mathrm{~A}$ & $2.48 \%$ & 0.0014 & $1.48 \%$ & 0.0008 & 11 \\
\hline \multirow[t]{11}{*}{$\mathrm{P}=0.1524$} & GCCTGGG & $* 5 \mathrm{~B}$ & $25.38 \%$ & 0.0015 & $32.89 \%$ & 0.0008 & 210 \\
\hline & GCCCGGG & $* 5 \mathrm{C}$ & $0.82 \%$ & 0.0006 & $4.59 \%$ & 0.0005 & 26 \\
\hline & GCCCGAG & $* 5 \mathrm{D}$ & $0.00 \%$ & 0.0000 & $0.01 \%$ & 0.0005 & 0 \\
\hline & GTTCAAG & $* 6 \mathrm{~A}$ & $36.88 \%$ & 0.0008 & $33.11 \%$ & 0.0014 & 226 \\
\hline & GTTCAGG & $* 6 \mathrm{C}$ & $0.01 \%$ & 0.0007 & $0.62 \%$ & 0.0010 & 3 \\
\hline & GTTCGAA & $* 7 \mathrm{~B}$ & $5.74 \%$ & 0.0000 & $4.96 \%$ & 0.0001 & 34 \\
\hline & GCTCGGG & $* 12 \mathrm{~A}$ & $0.83 \%$ & 0.0013 & $0.69 \%$ & 0.0010 & 5 \\
\hline & GCTTGGG & $* 12 \mathrm{C}$ & $0.00 \%$ & 0.0005 & $0.00 \%$ & 0.0002 & 0 \\
\hline & GTTCGAG & $* 13 \mathrm{~A}$ & $0.00 \%$ & 0.0003 & $0.64 \%$ & 0.0009 & 3 \\
\hline & ATTCGAG & $* 14 \mathrm{~B}$ & $0.00 \%$ & 0.0000 & $0.09 \%$ & 0.0009 & 1 \\
\hline & ATTCAAG & $* 14 \mathrm{D}$ & $0.00 \%$ & 0.0000 & $0.09 \%$ & 0.0009 & 0 \\
\hline \multicolumn{8}{|l|}{ Pakistan } \\
\hline 106 cases & GCTCGAG & $* 4$ & $24.44 \%$ & 0.0000 & $25.47 \%$ & 0.0003 & 164 \\
\hline 225 controls & GCCTGAG & $* 5 \mathrm{~A}$ & $2.22 \%$ & 0.0001 & $4.25 \%$ & 0.0003 & 19 \\
\hline \multirow[t]{4}{*}{$\mathrm{P}=0.1591$} & GCCTGGG & $* 5 \mathrm{~B}$ & $39.78 \%$ & 0.0001 & $33.96 \%$ & 0.0003 & 251 \\
\hline & GCCCGGG & $* 5 \mathrm{C}$ & $1.78 \%$ & 0.0001 & $1.89 \%$ & 0.0000 & 12 \\
\hline & GTTCAAG & $* 6 \mathrm{~A}$ & $27.78 \%$ & 0.0001 & $33.02 \%$ & 0.0000 & 195 \\
\hline & GTTCGAA & $* 7 \mathrm{~B}$ & $4.00 \%$ & 0.0000 & $1.42 \%$ & 0.0000 & 21 \\
\hline \multicolumn{8}{|l|}{ Venezuela } \\
\hline 75 cases & GCTCGAG & $* 4$ & $27.07 \%$ & 0.0014 & $21.95 \%$ & 0.0018 & 136 \\
\hline 190 controls & GCCTGAG & $* 5 \mathrm{~A}$ & $2.15 \%$ & 0.0011 & $4.05 \%$ & 0.0018 & 14 \\
\hline \multirow[t]{10}{*}{$\mathrm{P}=0.0006$} & GCCTGGG & $* 5 \mathrm{~B}$ & $32.18 \%$ & 0.0018 & $45.95 \%$ & 0.0018 & 192 \\
\hline & GCCCGGG & $* 5 \mathrm{C}$ & $1.58 \%$ & 0.0002 & $2.67 \%$ & 0.0000 & 10 \\
\hline & GTCTGGG & $* 5 \mathrm{G}$ & $0.01 \%$ & 0.0005 & $0.00 \%$ & 0.0000 & 0 \\
\hline & GTTCAAG & $* 6 \mathrm{~A}$ & $22.37 \%$ & 0.0003 & $22.67 \%$ & 0.0003 & 119 \\
\hline & GTTCGAA & $* 7 \mathrm{~B}$ & $9.47 \%$ & 0.0003 & $2.67 \%$ & 0.0002 & 40 \\
\hline & GCTCGGG & $* 12 \mathrm{~A}$ & $2.41 \%$ & 0.0012 & $0.05 \%$ & 0.0018 & 9 \\
\hline & GTTCGAG & $* 13 \mathrm{~A}$ & $2.50 \%$ & 0.0014 & $0.00 \%$ & 0.0002 & 9 \\
\hline & ATTCGAG & $* 14 \mathrm{~B}$ & $0.13 \%$ & 0.0013 & $0.00 \%$ & 0.0000 & 1 \\
\hline & ACCTGGG & $* 14 \mathrm{C}$ & $0.13 \%$ & 0.0013 & $0.00 \%$ & 0.0000 & 0 \\
\hline & ATCTGGG & $* 14 \mathrm{E}$ & $0.00 \%$ & 0.0003 & $0.00 \%$ & 0.0000 & 0 \\
\hline
\end{tabular}

E(Freq): Estimated frequency, S.E.: Standard error,P value: Permutation test $\mathrm{P}$ value testing for differences in haplotype frequencies between cases and controls (10,000 iterations)

Table 5. Elevated bladder cancer risk can only be confirmed for Venezuelan slow acetylators

\begin{tabular}{|c|c|c|c|c|c|c|c|c|}
\hline Acetylation status & Germany & & Hungary & & Pakistan & & Venezuela & \\
\hline & Cases & Controls & Cases & Controls & Cases & Controls & Cases & Controls \\
\hline Slow & $59.15 \%$ & $59.55 \%$ & $59.56 \%$ & $50.82 \%$ & $53.77 \%$ & $56.00 \%$ & $58.67 \%$ & $45.26 \%$ \\
\hline Rapid & $40.85 \%$ & $40.45 \%$ & $40.44 \%$ & $49.18 \%$ & $46.23 \%$ & $44.00 \%$ & $41.33 \%$ & $54.74 \%$ \\
\hline $\mathrm{N}$ & 781 & 1,931 & 272 & 61 & 106 & 225 & 75 & 190 \\
\hline Chi-square test $\mathrm{P}$ & 0.8478 & & 0.2114 & & 0.7039 & & 0.0493 & \\
\hline OR & 0.98 & & 1.43 & & 0.91 & & 1.72 & \\
\hline $95 \% \mathrm{CI}$ & $0.83-1.16$ & & $0.82-2.49$ & & $0.57-1.45$ & & $0.999-2.95$ & \\
\hline
\end{tabular}

All combined: $\mathrm{P}=0.5617 ; \mathrm{OR}=1.04 ; 95 \% \mathrm{CI}=0.90-1.21$, using the method of Mantel-Haenszel

ambiguity clarification data from different sources, i.e. populations, case-control groups, can be pooled together to obtain a higher precision of the estimates due to a larger sample size and more unambiguous information as long as the haplotype distribution differs not extremely between the populations.
Meanwhile, there are several statistical algorithms and programs available enabling a rapid and accurate haplotype prediction (73-76; for review see 77, 78) though PHASE v2.1 $(59,60)$ seems to yield consistently excellent results $(62,79-82)$. The use of 
haplotype reconstruction algorithms has become a standard solution in phase assignment, especially for NAT2 $(49,52-$ 56, 83-85). Furthermore, programs to derive the NAT2 haplotypes from unphased genotypes are publicly available, e.g. $(86,87)$.

\subsection{Haplotype distribution}

The present case-control series showed clear differences in the haplotype distribution between the study groups. Remarkably, the variability between the control groups was as or even more prominent as the differences between bladder cancer cases and controls. This is in accordance with the findings of most studies including different ethnicities or populations from the same ethnic group. Garcia-Martin, for instance, found in a metaanalysis of the seven most common NAT2 SNPs notable intra-ethnic differences for most SNPs not only in African or Asian study populations but also in Caucasians from the same geographic region (50).

In the Hungarian controls a remarkable low frequency of the $* 5 \mathrm{~B}(25 \%)$ and quite high frequency of *6A (3/\%) haplotypes was observed (Table 2) that could not be explained by comparison with geographically nearby populations. Mrozikiewicz et al. (88) found in a Polish study group haplotype frequencies quite similar to Caucasian study groups though also with a lower frequency of $* 5 \mathrm{~B}(33 \%)$ similar to Romanians $(34 \%)(85)$ - a trend that was not observed in Russians (37\%) (89), Czechs $(39 \%)$ and Greeks (38\%) (51).

Remarkably the study group from Pakistan exhibits a NAT2 haplotype distribution very similar to Caucasian study groups from Western and Central Europe. In fact, Pakistanis belong to those Central and South Asian populations that are considered as Indo-Europeans. Generally, NAT2 haplotypes are highly variable in Central Asian populations $(51,53)$. Sabbagh et al. conclude that Central and South Asian populations exhibit a haplotype distribution in between Europeans and East Asians with a higher frequency of $* 7 \mathrm{~B}$ and a virtually absence of $* 5 \mathrm{~B}-\mathrm{a}$ trend that could not be confirmed in the present Pakistani group (49).

The Venezuelan study group deviated most with respect to its haplotype distribution with several haplotypes being quite similar to the Spanish cohort, e.g. the occurrence of the rare $* 14$ haplotypes which is debated to be due to an admixture of African descendants in the Spanish population (50). The occurrence of the rare *14 haplotypes is also common in South American populations due to South-West European, especially Spanish and Portuguese, descendants $(49,72,90,91)$. Jorge-Nebert et al. (52) found in a study of Amerindians from Panama besides over $60 \% * 4$ haplotypes, over $20 \% * 7 \mathrm{~B}$ haplotypes that are also more frequent in our Venezuelan study group (Table 2) but not in Amerindian and admixed populations from Brazil $(72,92)$. This high frequency of $* 7 \mathrm{~B}$ haplotypes was also reported by Fuselli et al. (90) generally for American populations with a high intra-ethnic variability and by Sabbagh et al. (49) for Asian and Central American populations. Furthermore, Jorge-Nebert et al.
(52) observe a lower frequency of the slow genotypes than typical for Caucasians $(>50 \%)$ which is more similar to Asians and other Amerindians $(<30 \%)(51,52,90)$. The *14 haplotypes were also observed quite often in an admixed Brazilian population, in particular in Brazilians of Caucasian and African descent (72). Native Latin Americans showed different haplotype distribution patterns resulting in a much higher percentage of rapid acetylators (75\%) (90). Teixeira et al. observed in their two Brazilian study groups considerable differences in the haplotype distribution especially for the $* 4$ haplotype (92). Their mainly Amerindian/European study group was quite similar to our Venezuelan group besides the more frequent occurrence of the *7B haplotype in our sample. The *6A haplotype - less frequent in the Venezuelan study group was almost absent in the Amerindians from Panama (52) but showed a frequency similar to Caucasians in the Brazilian study groups of Teixeira et al. (92) while Talbot et al. (72) reported lower $* 6 \mathrm{~B}$ frequencies. Fuselli et al. discussed the occurrence of $* 6$ haplotypes in native Americans to be solely due to an European or African admixture (90). Thus, the results of the Venezuelan study group which was recruited from the general population with no special focus on native Americans were in accordance with an admixed South-American population with a considerable part of Spanish ancestry.

\subsection{Haplotype implications}

Most studies consider the mutation sites alone. This hampers comparisons of haplotype frequencies, linkage disequilibrium patterns and distributions of slow, intermediate and rapid acetylators. As rapid, reliable and easy-to-handle haplotype reconstruction algorithms are meanwhile standard tools which allow to pool data from different populations - if they are not too distinct and represented by a sufficient number of samples - we strongly recommend their use to provide valuable genetic information.

Reconstructing NAT2 haplotypes circumvents for the main body of the data the insecurity about the acetylation status assignment as only one instead of several haplotype pairs has to be considered. Furthermore, it provides valuable information about the chromosomal phase which may - in addition to the acetylation status as potential risk factor regarded in most epidemiological studies - help to clarify and understand the role of NAT2 in the development of some cancers. In particular, it is wellknown from in vitro assays that NAT2 haplotypes differ in stability, enzymatic activity and substrate affinity (1, 45, 48,93 ). Hence different impacts of the NAT2 haplotypes on the individual susceptibility for some cancers and other diseases via altered metabolic effects are discussed $(25,29$, $45,47)$.

For the present study groups conclusions on the haplotype basis are difficult due to sample size limitations. Only the German study group is large enough so that differences in haplotype distributions, e.g. between smoking and non-smoking cases might yield interesting results. Remarkably, the Venezuelan bladder cancer cases showed a clearly elevated frequency of the $* 5 \mathrm{~B}$ haplotype that is debated to be extremely slow (48). 


\subsection{Recent advances in NAT2 phenotype prediction: Reducing the genotype to one or two SNPS}

Recently, a novel NAT2 tagSNP (rs1495741) has been identified that predicts the seven SNP inferred NAT2 phenotype with high accuracy $(24,64)$. Garcia-Closas et al. compared rs1495741 genotypes to NAT2 activity measured in lysates of cryopreserved human hepatocytes (64). They conclude that the novel NAT2 tagSNP predicts with high accuracy the NAT2 phenotype and can be used as a sole marker in populations with European background (64). Selinski et al. confirmed the general good performance of this tagSNP with respect to 3,177 NAT2 and tagSNP genotypes of European, Venezuelan and Pakistani study groups with minor ethnic differences between both genotypic markers predicting the acetylation capacity (94). Furthermore they found a higher specificity of the NAT2 genotype with respect to in vivo phenotypes of 344 Germans measured by the caffeine test $(95,96)$ though the sensitivity of the tagSNP was indeed excellent but the latter mispredicted several rapid acetylators as slow. In addition, they suggested alternatively a 2-SNP combination (C282T, T341C; rs1041983, rs1801280) for simple acetylation velocity prediction that yielded almost the same results as the NAT2 genotype. This combination was already suggested in 1995 by Cascorbi et al. and in 2003 by Agundez showing excellent results also for Chinese and Japanese study groups $(29,41,97)$. In the present data set the haplotypes that would be misclassified by the 2-SNP genotype as rapid $(* 6 \mathrm{~B}$ : total $0.04 \%$, Spain $2 \%$; $* \mathrm{E}$ : not present; *6F: not present; *7A: not present; *14A: total $0.02 \%$, Spain $0.4 \%$; *14E: Spain $0.04 \%$, *14I: Spain $0.04 \%$; see Table 2 ) or slow (*13A: total $0.3 \%$, Spain $0.3 \%$ ) are also quite rare.

Comparing these findings to the 28 study groups from different populations and ethnicities from Sabbagh et al. (49) (in total 7,988 haplotypes) yielded $0.7 \%$ haplotypes that would be mispredicted as rapid by the 2-SNP genotype and $1.3 \%$ of the haplotypes being mispredicted as slow. In particular, the slow and rare $* 6 \mathrm{~B}$ was found in some African populations, Spaniards and UK Caucasians, *7A was present in one African and the Korean study group and *14A was observed in the African, Spanish and Nicaraguan study groups whereas the rapid *13A haplotype was quite frequent in the African study groups (about 4-10\%) and also found in Europeans and East Asians.

Thus, for minimizing NAT2 genotyping effort the use of the tagSNP rs1495741 (64) or the 2-SNP genotype (C282T, T341C) (94) as phenotype predictors might be reasonable as long as no potential substrate-specific differences between NAT2 haplotypes have to be taken into account or ethnic differences and limited studies demand a careful use. A reduction of genotyping effort would especially be helpful in individualised dosing depending on NAT2 activity, for instance in anti-tuberculosis therapy and in larger epidemiological studies, for instance evaluating individual susceptibility to bladder carcinogens $(7,8,19)$.

\subsection{Summary}

In brief, haplotype reconstruction yielded consistent results across different populations and study groups enabling the analysis of haplotype frequencies with high reliability. We confirmed intra-ethnic differences within European populations with the Hungarian study group differing most while the Pakistani study group was quite similar to the German and Spanish controls. The main differences between the study groups could be observed for the slow haplotypes $* 5 \mathrm{~B}$ that was lowest in Hungarian controls $(25 \%$ vs. $32-43 \%)$, *6A that was highest in Hungarian (37\%) and lowest in Venezuelan controls (22\%) and $* 7 \mathrm{~B}$ that was highest in Venezuelan controls $(9 \%)$. The rare *14 haplotypes were found in Spaniards and Venezuelans but also in Germans. The supposed effect of slow NAT2 genotypes on bladder cancer could only be confirmed for the Venezuelan study group with a lower frequency of rapid $* 4(22 \%$ vs. $27 \%)$ and a higher frequency of slow $* 5 \mathrm{~B}(46 \% v s$. $32 \%)$ haplotypes in the cases. The not significant results for the three other bladder cancer case-control series are in accordance with current studies and meta-analysis that show small effects of NAT2 if smoking habits and occupational exposure to bladder carcinogens are not taken into account.

\section{ACKNOWLEDGEMENTS}

The authors thank Ms Doris Dannappel, Ms Marion Page, Ms Kirsten Liesenhoff-Henze, for excellent technical support.

\section{REFERENCES}

1. DW Hein: N-Acetyltransferase SNPs: emerging concepts serve as a paradigm for understanding complexities of personalized medicine. Expert Opin Drug Metab Toxicol 5, 353-366 (2009)

2. DW Hein: Molecular genetics and function of NAT1 and NAT2: role in aromatic amine metabolism and carcinogenesis. Mutat Res 506-507, 65-77 (2002)

3. DW Hein: N-Acetyltransferase genetics and their role in predisposition to aromatic and heterocyclic amine-induced carcinogenesis. Toxicol Lett 112-113, 349-56 (2000)

4. HB Hughes, JB Biehl, AP Jones, LH Schmidt: Metabolism of isoniazid in man as related to the occurrence of peripheral neuritis. Am Rev Tuberc 70, 266-273 (1954)

5. RS Mitchell, JC Bell: Clinical implications of isoniazid blood levels in pulmonary tuberculosis. N Engl J Med 257, 1066-1070 (1957)

6. LE Moore, DR Baris, JD Figueroa, M Garcia-Closas, MR Karagas, MR Schwenn, AT Johnson, JH Lubin, DW Hein, CL Dagnall, JS Colt, M Kida, MA Jones, AR Schned, SS Cherala, SJ Chanock, KP Cantor, DT Silverman, N Rothman: GSTM1 null and NAT2 slow acetylation genotypes, smoking intensity and bladder cancer risk: results from the New England bladder cancer study and NAT2 meta-analysis. Carcinogenesis 32, 182-189 (2011)

7. RG Hall, RD Leff, T Gumbo: Treatment of active pulmonary tuberculosis in adults: current standards and 
recent advances. Insights from the Society of Infectious Diseases Pharmacists. Pharmacotherapy 29, 1468-1481 (2009)

8. K Fukino, Y Sasaki, S Hirai, $\mathrm{T}$ Nakamura, M Hashimoto, F Yamagishi, K Ueno: Effects of Nacetyltransferase 2 (NAT2), CYP2E1 and glutathione-Stransferase (GST) genotypes on the serum concentrations of isoniazid and metabolites in tuberculosis patients. $J$ Toxicol Sci 33, 187-195 (2008)

9. W Weistenhöfer, M Blaszkewicz, HM Bolt, K Golka: $\mathrm{N}$-Acetyltransferase-2 and medical history in bladder cancer cases with a suspected occupational disease (BK 1301) in Germany. J Toxicol Environ Health A 71, 906910 (2008)

10. K Golka, T Seidel, H Dietrich, G Roth, C Rötzel, R Thier, F Geller, T Reckwitz, H Schulze: Occupational and non-occupational risk factors in bladder cancer patients in an industrialized area located in former EastGermany. Aktuelle Urol 36, 417-422 (2005)

11. K Golka, V Prior, M Blaszkewicz, HM Bolt: The enhanced bladder cancer susceptibility of NAT2 slow acetylators towards aromatic amines: a review considering ethnic differences. Toxicol Lett 2002 128, 229-241 (2002)

12. K Golka, W Weistenhöfer, P Jedrusik, F Geller, M Blaszkewicz, HM Bolt: N-Acetyltransferase 2 phenotype in painters with bladder cancer and controls. Ann Acad Med Singapore 30, 464-467 (2001)

13. K Golka, T Reckwitz, M Kempkes, I Cascorbi, M Blaskewicz, SE Reich, I Roots, J Soekeland, H Schulze, HM Bolt: N-Acetyltransferase 2 (NAT2) and glutathione S-transferase $\mu$ (GSTM1) in bladder-cancer patients in a highly industrialized area. Int J Occup Environ Health 3, 105-110 (1997)

14. K Golka, V Prior, M Blaszkewicz, I Cascorbi, W Schöps, G Kierfeld, I Roots, HM Bolt: Occupational history and genetic $\mathrm{N}$-acetyltransferase polymorphism in urothelial cancer patients of Leverkusen, Germany. Scand J Work Environ Health 22, 332-338 (1996)

15. M Garcia-Closas, N Malats, D Silverman, M Dosemeci, M Kogevinas, DW Hein, A Tardon, C Serra, A Carrato, R Garcia-Closas, J Lloreta, G CastañoVinyals, M Yeager, R Welch, S Chanock, N Chatterjee, $\mathrm{S}$ Wacholder, C Samanic, M Torà, F Fernández, FX Real, N Rothman: NAT2 slow acetylation, GSTM1 null genotype, and risk of bladder cancer: results from the Spanish Bladder Cancer Study and meta-analyses. Lancet 366, 649-659 (2005)

16. RJ Hung, P Boffetta, P Brennan, C Malaveille, A Hautefeuille, F Donato, U Gelatti, M Spaliviero, D Placidi, A Carta, A Scotto di Carlo, S Porru: GST, NAT, SULT1A1, CYP1B1 genetic polymorphisms, interactions with environmental exposures and bladder cancer risk in a high-risk population. Int J Cancer 110, 598-604 (2004)

17. QW Ma, GF Lin, JG Chen, CQ Xiang, WC Guo, K Golka, JH Shen: Polymorphism of N-acetyltransferase 2 (NAT2) gene polymorphism in Shanghai population: occupational and non-occupational bladder cancer patient groups. Biomed Environ Sci 17, 291-298 (2004)

18. R Thier, T Brüning, PH Roos, HP Rihs, K Golka, Y Ko, HM Bolt: Markers of genetic susceptibility in human environmental hygiene and toxicology: the role of selected CYP, NAT and GST genes. Int J Hyg Environ Health 206, 149-171 (2003)

19. YS Huang, HD Chern, WJ Su, JC Wu, SL Lai, SY Yang, FY Chang, SD Lee: Polymorphism of the Nacetyltransferase 2 gene as a susceptibility risk factor for antituberculosis drug-induced hepatitis. Hepatology 35, 883-889 (2002)

20. P Vineis, D Marinelli, H Autrup, J Brockmöller, I Cascorbi, AK Daly, K Golka, H Okkels, A Risch, N Rothman, E Sim, E Taioli: Current smoking, occupation, $\mathrm{N}$-acetyltransferase- 2 and bladder cancer: a pooled analysis of genotype-based studies. Cancer Epidemiol Biomarkers Prev 10, 1249-1252 (2001)

21. JG Hengstler, $M$ Arand, ME Herrero, $F$ Oesch: Polymorphisms of $\mathrm{N}$-acetyltransferases, glutathione $\mathrm{S}$ transferases, microsomal epoxide hydrolase and sulfotransferases: influence on cancer susceptibility. Recent Results Cancer Res 154, 47-85 (1998)

22. JA Agundez: Polymorphisms of human Nacetyltransferases and cancer risk. Curr Drug Metab 9, 520-531 (2008)

23. DW Hein: N-Acetyltransferase 2 genetic polymorphism: effects of carcinogen and haplotype on urinary bladder cancer risk. Oncogene 25, 1649-1658 (2006)

24. N Rothman, M Garcia-Closas, N Chatterjee, N Malats, X Wu, JD Figueroa, FX Real, D Van Den Berg, G Matullo, D Baris, M Thun, LA Kiemeney, P Vineis, I De Vivo, D Albanes, MP Purdue, T Rafnar, MA Hildebrandt, AE Kiltie, O Cussenot, K Golka, R Kumar, JA Taylor, JI Mayordomo, KB Jacobs, M Kogevinas, A Hutchinson, Z Wang, YP Fu, L Prokunina-Olsson, L Burdett, M Yeager, W Wheeler, A Tardón, C Serra, A Carrato, R GarciaClosas, J Lloreta, A Johnson, M Schwenn, MR Karagas, A Schned, G Andriole Jr, R Grubb III, A Black, EJ Jacobs, WR Diver, SM Gapstur, SJ Weinstein, J Virtamo, VK Cortessis, M Gago-Dominguez, MC Pike, MC Stern, JM Yuan, DJ Hunter, M McGrath, CP Dinney, B Czerniak, M Chen, H Yang, SH Vermeulen, KK Aben, JA Witjes, RR Makkinje, P Sulem, S Besenbacher, K Stefansson, E Riboli, P Brennan, S Panico, C Navarro, NE Allen, HB Bueno-de-Mesquita, D Trichopoulos, N Caporaso, MT Landi, F Canzian, B Ljungberg, A Tjonneland, F ClavelChapelon, DT Bishop, MT Teo, MA Knowles, S Guarrera, 
S Polidoro, F Ricceri, C Sacerdote, A Allione, G CancelTassin, S Selinski, JG Hengstler, H Dietrich, T Fletcher, $\mathrm{P}$ Rudnai, E Gurzau, K Koppova, SC Bolick, A Godfrey, Z $\mathrm{Xu}$, JI Sanz-Velez, MD Garcia-Prats, M Sanchez, G Valdivia, S Porru, S Benhamou, RN Hoover, JF Fraumeni Jr, DT Silverman, SJ Chanock: A multi-stage genome-wide association study of bladder cancer identifies multiple susceptibility loci. Nat Genet 42, 978-984 (2010)

25. JM Walraven, $\mathrm{Y}$ Zang, JO Trent, DW Hein: Structure/function evaluations of single nucleotide polymorphisms in human $\mathrm{N}$-acetyltransferase 2. Curr Drug Metab 9, 471-486 (2008)

26. DM Grant: Structures of human arylamine Nacetyltransferases. Curr Drug Metab 9, 465-70 (2008)

27. DM Grant: Molecular genetics of the Nacetyltransferases. Pharmacogenetics 3, 45-50 (1993)

28. DM Grant, M. Blum and U.A. Meyer: Polymorphisms of N-acetyltransferase genes. Xenobiotica 22, 1073-1081 (1992)

29. I Cascorbi, I Roots: Pitfalls in N-acetyltransferase 2 genotyping. Pharmacogenetics 9, 123-127 (1999)

30. DW Hein, MA Doll, TD Rustan, RJ Ferguson: Metabolic activation of N-hydroxyarylamines and $\mathrm{N}$ hydroxyarylamides by 16 recombinant human NAT2 allozymes: effects of 7 specific NAT2 nucleic acid substitutions. Cancer Res 55, 3531-3536 (1995)

31. DW Hein, RJ Ferguson, MA Doll, TD Rustan, K Gray: Molecular genetics of human polymorphic Nacetyltransferase: enzymatic analysis of 15 recombinant wild-type, mutant and chimeric NAT2 allozymes. Hum Mol Genet 3, 729-734 (1994)

32. DW Hein, MA Doll, TD Rustan, K Gray, Y Feng, RJ Ferguson, DM Grant: Metabolic activation and deactivation of arylamine carcinogens by recombinant human NAT1 and polymorphic NAT2 acetyltransferases. Carcinogenesis 14, 1633-1638 (1993)

33. KPVatsis, WW Weber, DA Bell, JM Dupret, DAP Evans, DM Grant, DW Hein, HJ Lin, UA Meyer, MV Relling, E Sim, T Suzuki, Y Yamazoe: Nomenclature for $\mathrm{N}$-acetyltransferases. Pharmacogenetics 5, 1-17 (1995)

34. DA Bell, JA Taylor, MA Butler, EA Stephens, J Wiest, LH Brubaker, FF Kadlubar, GW Lucier: Genotype/phenotype discordance for human arylamine $\mathrm{N}$-acetyltransferase (NAT2) reveals a new slowacetylator allele common in African-Americans. Carcinogenesis 14, 1689-1692 (1993)

35. M Blum, A Demierre, DM Grant, M Heim, UA Meyer: Molecular mechanism of slow acetylation of drugs and carcinogens in humans. Proc Natl Acad Sci U $S$ A 88, 5237-41 (1991)

36. D Hickman, E Sim: N-Acetyltransferase polymorphism. Comparison of phenotype and genotype in humans. Biochem Pharmacol 42, 1007-1014 (1991)

37. JA Agundez, K Golka, C Martinez, S Selinski, M Blaszkewicz, E Garcia-Martin: Unraveling ambiguous NAT2 genotyping data. Clin Chem 54, 1390-1394 (2008)

38. K Golka, M Blaszkewicz, M Samimi, HM Bolt, S Selinski: Reconstruction of N-acetyltransferase 2 haplotypes using PHASE. Arch Toxicol 82, 265-270 (2008)

39. HM Bolt, S Selinski, D Dannappel, M Blaszkewicz, K Golka: Re-investigation of the concordance of human NAT2 phenotypes and genotypes. Arch Toxicol 79, 196200 (2008)

40. AC Deitz, N Rothman, TR Rebbeck, RB Hayes, WH Chow, W Zheng, DW Hein, M Garcia-Closas: Impact of misclassification in genotype-exposure interaction studies: example of N-acetyltransferase 2 (NAT2), smoking, and bladder cancer. Cancer Epidemiol Biomarkers Prev 13, 1543-1546 (2004)

41. I Cascorbi, N Drakoulis, J Brockmöller, A Maurer, K Sperling, I Roots: Arylamine N-acetyltransferase (NAT2) mutations and their allelic linkage in unrelated Caucasian individuals: correlation with phenotypic activity. Am J Hum Genet 57, 581-592 (1995)

42. DW Hein, DM Grant, E Sim: Update on consensus arylamine $\mathrm{N}$-acetyltransferase gene nomenclature. Pharmacogenetics 10, 291-292 (2000)

43. DW Hein, S Boukouvala, DM Grant, RF Minchin, E Sim: Changes in consensus arylamine $\mathrm{N}$-acetyltransferase gene nomenclature. Pharmacogenet Genomics 18, 367-368 (2008)

44. Arylamine N-acetyltransferase Nomenclature Committee. Update November 1, 2010. http://www.louisville.edu/medschool/pharmacology/NAT.h tml (accessed Feb 2, 2011)

45. Y Zang, MA Doll, S Zhao, JC States, DW Hein: Functional characterization of single-nucleotide polymorphisms and haplotypes of human Nacetyltransferase 2. Carcinogenesis 28, 1665-1671 (2007)

46. DW Hein, MA Doll, AJ Fretland, MA Leff, SJ Webb, GH Xiao, US Devanaboyina, NA Nangju, Y Feng: Molecular genetics and epidemiology of the NAT1 and NAT2 acetylation polymorphisms. Cancer Epidemiol Biomarkers Prev 9, 29-42 (2000)

47. JA Agundez, JG Menaya, R Tejeda, F Lago, M Chavez, J Benitez: Genetic analysis of the NAT2 and CYP2D6 polymorphisms in white patients with non-insulin- 
dependent diabetes mellitus. Pharmacogenetics 6, 465-472 (1996)

48. MA Leff, AJ Fretland, MA Doll, DW Hein: Novel human $\mathrm{N}$-acetyltransferase 2 alleles that differ in mechanism for slow acetylator phenotype. $J$ Biol Chem 274, 34519-34522 (1999)

49. A Sabbagh, A Langaney, P Darlu, N Gerard, R Krishnamoorthy, ES Poloni: Worldwide distribution of NAT2 diversity: implications for NAT2 evolutionary history. BMC Genet 9, 21 (2008)

50. E Garcia-Martin: Interethnic and intra-ethnic variability of NAT2 single nucleotide polymorphisms. Curr Drug Metab 9, 487-497 (2008)

51. F Luca, G Bubba, $M$ Basile, $\mathrm{R}$ Brdicka, E Michalodimitrakis, O Rickards, G Vershubsky, L Quintana-Murci, AI Kozlov, A Novelletto: Multiple advantageous amino acid variants in the NAT2 gene in human populations. PLoS One 3, e3136 (2008)

52. LF Jorge-Nebert, $M$ Eichelbaum, EU Griese, $T$ Inaba, TD Arias: Analysis of six SNPs of NAT2 in Ngawbe and Embera Amerindians of Panama and determination of the Embera acetylation phenotype using caffeine. Pharmacogenetics 12, 39-48 (2002)

53. H Magalon, E Patin, F Austerlitz, T Hegay, A Aldashev, L Quintana-Murci, E Heyer: Population genetic diversity of the NAT2 gene supports a role of acetylation in human adaptation to farming in Central Asia. Eur J Hum Genet 16, 243-251 (2008)

54. E Patin, LB Barreiro, PC Sabeti, F Austerlitz, F Luca, A Sajantila, DM Behar, O Semino, A Sakuntabhai, N Guiso, B Gicquel, K McElreavey, RM Harding, E Heyer, L Quintana-Murci: Deciphering the ancient and complex evolutionary history of human arylamine N-acetyltransferase genes. Am J Hum Genet $78,423-436$ (2006)

55. E Patin, C Harmant, KK Kidd, J Kidd, A Froment, SQ Mehdi, L Sica, E Heyer, L Quintana-Murci: SubSaharan African coding sequence variation and haplotype diversity at the NAT2 gene. Hum Mutat 27, 720 (2006)

56. A Sabbagh, P Darlu: Inferring haplotypes at the NAT2 locus: the computational approach. BMC Genet 6 , 30 (2005)

57. M Blaszkewicz, D Dannappel, R Thier, J Lewalter: $\mathrm{N}$-Acetyltransferase 2 (genotyping). In: Analyses of hazardous substances in biological materials, vol 9 . Special issue: Markers of susceptibility. Eds: J Angerer, M Müller, T Weiss et al. Eds. Wiley-VCH, Weinheim, Germany (2004)

58. I Cascorbi, J Brockmöller, PM Mrozikiewicz, S Bauer, R Loddenkemper, I Roots: Homozygous rapid arylamine N-acetyltransferase NAT2 genotype as susceptibility factor for lung cancer. Cancer Res 56, 39613966 (1996)

59. M Stephens, NJ Smith, P Donnelly: A new statistical method for haplotype reconstruction from population data. Am J Hum Genet 68, 978-89 (2001)

60. M. Stephens and P. Donnelly: A comparison of Bayesian methods for haplotype reconstruction. Am J Hum Genet 73, 1162-1169 (2003)

61. M Stephens, P Scheet: Accounting for decay of linkage disequilibrium in haplotype inference and missing-data imputation. Am J Hum Genet 76, 449-462 (2005)

62. J Marchini, D Cutler, N Patterson, M Stephens, E Eskin, E Halperin, S Lin, ZS Qin, HM Munro, GR Abecasis, P Donnelly and the International HapMap Consortium: A comparison of phasing algorithms for trios and unrelated individuals. Am J Hum Genet 78, 437-450 (2006)

63. N Li, M Stephens: Modeling linkage disequilibrium and identifying recombination hotspots using singlenucleotide polymorphism data. Genetics 165 , 2213-2233 (2003)

64. M Garcia-Closas, DW Hein, D Silverman, N Malats, M Yeager, K Jacobs, MA Doll, JD Figueroa, D Baris, M Schwenn, M Kogevinas, A Johnson, N Chatterjee, LE Moore, T Moeller, FX Real, S Chanock, N Rothman: A single nucleotide polymorphism tags variation in the arylamine $\mathrm{N}$-acetyltransferase 2 phenotype in populations of European background. Pharmacogenet Genomics 21, 231-236 (2011)

65. P Boffetta: Tobacco smoking and risk of bladder cancer. Scand J Urol Nephrol Suppl 42(S218), 45-54 (2008)

66. MP Zeegers, RA Goldbohm, PA Van Den Brandt: A prospective study on active and environmental tobacco smoking and bladder cancer risk. Cancer Causes Control 13, 83-90 (2002)

67. MP Zeegers, FE Tan, E Dorant, PA Van Den Brandt: The impact of characteristics of cigarette smoking on urinary tract cancer risk: a meta-analysis of epidemiologic studies. Cancer 89, 630-639 (2000)

68. GL Delclos, SP Lerner: Occupational risk factors. Scandinavian Journal of Urology and Nephrology Suppl 42(S218), 58-63 (2008)

69. RC Reulen, E Kellen, F Buntinx, M Brinkman, MP Zeegers: A meta-analysis on the association between bladder cancer and occupation. Scandinavian Journal of Urology and Nephrology Suppl 42(S218), 64-78 (2008)

70. I Cascorbi, I Roots, J Brockmöller: Association of NAT1 and NAT2 polymorphisms to urinary bladder 
cancer: significantly reduced risk in subjects with NAT1*10. Cancer Res 61, 5051-5056 (2001)

71. A Risch, DM Wallace, S Bathers, E Sim: Slow Nacetylation genotype is a susceptibility factor in occupational and smoking related bladder cancer. Hum Mol Genet 4, 231-236 (1995)

72. J Talbot, LA Magno, CV Santana, SM Sousa, PR Melo, RX Correa, G Di Pietro, F Rios-Santos: Interethnic diversity of NAT2 polymorphisms in Brazilian admixed populations. BMC Genet 11, 87 (2010)

73. L Excoffier, G Laval, S Schneider: Arlequin ver. 3.0: An integrated software package for population genetics data analysis. Evolutionary Bioinformatics Online 1, 47-50 (2005)

74. P Scheet, M Stephens: A fast and flexible statistical model for large-scale population genotype data: applications to inferring missing genotypes and haplotypic phase. Am J Hum Genet 78, 629-644 (2006)

75. T Niu, ZS Qin, X Xu, JS Liu: Bayesian haplotype inference for multiple linked single-nucleotide polymorphisms. Am J Hum Genet 70, 157-169 (2002)

76. ZS Qin, T Nui, JS Liu: Partition-ligation-expectationmaximization algorithm for haplotype inference with single-nucleotide polymorphisms. Am J Hum Genet 71, 1242-1247 (2002)

77. RM Salem, J Wessel, NJ Schork: A comprehensive literature review of haplotyping software and methods for use with unrelated individuals. Hum Genomics 2, 39-66 (2005)

78. T Niu: Algorithms for inferring haplotypes. Genet Epidemiol 27, 334-347 (2004)

79. C Coulonges, O Delaneau, M Girard, H Do, R Adkins, J-L Spadoni, J-F Zagury: Computation of haplotypes on SNPs subsets: advantage of the "global method". BMC Genet 7, 50 (2006)

80. L Eronen, F Geerts, H Toivonen: HaploRec: effcient and accurate large-scale reconstruction of haplotypes. BMC Bioinformatics 7, 542 (2006)

81. J Zhang, M Vingron, MR Hoehe: Haplotype reconstruction for diploid populations. Hum Hered 59, 144$156(2005)$

82. RM Adkins: Comparison of the accuracy of methods of computational haplotype inference using a large empirical dataset. BMC Genet 5, 22 (2004)

83. M Jain, S Kumar, P Lal, A Tiwari, UC Ghoshal, B Mittal: Association of genetic polymorphisms of $\mathrm{N}$ acetyltransferase 2 and susceptibility to esophageal cancer in north Indian population. Cancer Invest 25, 340-346 (2007)
84. SC Sak, JH Barrett, AB Paul, DT Bishop, AE Kiltie: DNA repair gene XRCC1 polymorphisms and bladder cancer risk. BMC Genet 8, 13 (2007)

85. S Rabstein, K Unfried, U Ranft, T Illig, M Kolz, HP Rihs, C Mambetova, M Vlad, T Brüning, B Pesch: Variation of the $\mathrm{N}$-acetyltransferase 2 gene in a Romanian and a Kyrgyz population. Cancer Epidemiol Biomarkers Prev 15, 138-141 (2006)

86. IB Kuznetsov, M McDuffie, R Moslehi: A web server for inferring the human $\mathrm{N}$-acetyltransferase-2 (NAT2) enzymatic phenotype from NAT2 genotype. Bioinformatics $25,1185-1186$ (2009)

87. A Sabbagh, $\mathrm{P}$ Darlu, $\mathrm{M}$ Vidaud: Evaluating NAT2PRED for inferring the individual acetylation status from unphased genotype data. BMC Med Genet 10, 148 (2009)

88. PM Mrozikiewicz, I Cascorbi, J Brockmöller, I Roots: Determination and allelic allocation of seven nucleotide transitions within the arylamine $\mathrm{N}$-acetyltransferase gene in the Polish population. Clin Pharmacol Ther 59, 376-382 (1996)

89. EA Gaikovitch, I Cascorbi, PM Mrozikiewicz, J Brockmöller, R Frötschl, K Köpke, T Gerloff, JN Chernov, I Roots: Polymorphisms of drug-metabolizing enzymes CYP2C9, CYP2C19, CYP2D6, CYP1A1, NAT2 and of Pglycoprotein in a Russian population. Eur J Clin Pharmacol 59, 303-312 (2003)

90. S Fuselli, RH Gilman, SJ Chanock, SL Bonatto, G De Stefano, CA Evans, D Labuda, D Luiselli, FM Salzano, G Soto, G Vallejo, A Sajantila, D Pettener, E Tarazona-Santos: Analysis of nucleotide diversity of NAT2 coding region reveals homogeneity across Native American populations and high intra-population diversity. Pharmacogenomics J 7, 144152 (2007)

91. C Martinez, JA Agundez, M Olivera, A Llerena, R Ramirez, M Hernández, J Benitez: Influence of genetic admixture on polymorphisms of drug-metabolizing enzymes: analyses of mutations on NAT2 and C gamma P2E1 genes in a mixed Hispanic population. Clin Pharmacol Ther 63, 623-628 (1998)

92. RL Teixeira, AB Miranda, AG Pacheco, MQ Lopes, J Fonseca-Costa, MF Rabahi, HM Melo, AL Kritski, FC Mello, PN Suffys, AR Santos: Genetic profile of the arylamine Nacetyltransferase 2 coding gene among individuals from two different regions of Brazil. Mutat Res 624, 31-40 (2007)

93. L Le Marchand, L Sivaraman, AA Franke, LJ Custer, LR Wilkens, AF Lau, RV Cooney: Predictors of Nacetyltransferase activity: should caffeine phenotyping and NAT2 genotyping be used interchangeably in epidemiological studies? Cancer Epidemiol Biomarkers Prev 5, 449-455 (1996)

94. S Selinski, M Blaszkewicz, ML Lehmann, D Ovsiannikov, O Moormann, C Guballa, A Kress, MC Truß, H Gerullis, T Otto, D Barski, G Niegisch, P Albers, S 
Frees, W Brenner, JW Thüroff, M Angeli-Greaves, T Seidel, G Roth, H Dietrich, R Ebbinghaus, HM Prager, HM Bolt, M Falkenstein, A Zimmermann, T Klein, T Reckwitz, HC Roemer, D Löhlein, W Weistenhöfer, W Schöps, SAH Rizvi, M Aslam, G Banfi, I Romics, M Steffens, AB Ekici, A Winterpacht, K Ickstadt, H Schwender, JG Hengstler, K Golka: Genotyping NAT2 with only two SNPs (rs1041983 and rs1801280) outperforms the tagging SNP rs1495741 and is equivalent to the conventional 7-SNP NAT2 genotype. Pharmacogenet Genomics 21, 673-678 (2011)

95. DM Grant, BK Tang, W Kalow: A simple test for acetylator phenotype using caffeine. Br J Clin Pharmacol 17, 459-464 (1984)

96. M Blaszkewicz: N-Acetyltransferase 2 (phenotyping: caffeine test) In: Analyses of hazardous substances in biological materials, vol 9. Special issue: Markers of susceptibility. Eds: J Angerer, M Müller, T Weiss et al. Wiley-VCH, Weinheim, Germany (2004)

97. JAG Agundez: NAT2 genotyping: equilibrium between accuracy and feasibility in routine analyses. J Appl Res $3(2), 1-6(2003)$

Abreviations: CI: confidence interval, NAT2: Nacetyltransferase 2, OR: odds ratio, SNP: single nucleotide polymorphism

Key Words: rs1801279 (G191A), rs1041983 (C282T), rs1801280 (T341C), rs1799929 (C481T), rs1799930 (G590A), rs1208 (A803G), rs1799931 (G857A)

Send correspondence to: Silvia Selinski, Ardeystr. 67, 44129 Dortmund, Germany, Tel: 49-231-1084-216, Fax: 49-231-1084-343, E-mail: selinski@ifado.de 